\title{
Simple Channel Estimation Techniques Based on Pilot-Assistance for STBC-Based MIMO-OFDM Systems
}

\author{
Tran Duc Tan ${ }^{1}$, Ta Duc Tuyen ${ }^{1}$, Trinh Anh Vu ${ }^{1}$, Huynh Huu Tue ${ }^{2}$ \\ ${ }^{1}$ Faculty of Electronics and Telecommunications, College of Technology, VNUH \\ ${ }^{2} \mathrm{BacHa}$ International University Hanoi, Vietnam \\ Email: tantd@coltech.vnu.vn, tuyentd@vnu.edu.vn,vuta@vnu.edu.vn,huynhuutue@bhiu.edu.vn
}

\begin{abstract}
With equipping multiple antennas at both transmitter and receiver ends, the signals in the wireless OFDM systems could be transmitted over multipath fading channels for achieving benefits and high flexibility. In this paper, we focus on MIMO-OFDM systems that simultaneously perform channel estimation in order to combat additive noise, and multipath fading. The performance improvement is confirmed by simulations. Results show that the proposed MIMOOFDM scheme is quite robust in practical environments.
\end{abstract}

Keywords: Space-time block coder (STBC), MIMOOFDM systems, channel estimation

\section{INTRODUCTION}

Wireless systems have many advantages over its wired counterpart. However, the main difficulty of the wireless channel is multipath fading. Fading is interference caused by sum of two or more form of the transmitted signal. The combined signal arrives at the receiver antenna at slightly different times. This signal can vary widely in amplitude and phase. OFDM (Orthogonal Frequency Division Multiplexing) is a multi-carrier modulation scheme that has gained considerable popularity over the past decade because of its ability to combat frequency-selective fading normally encountered in a multi-path wireless environment. OFDM converts the frequency selective channel into flat-fading sub-channels, there by significantly reducing the receiver complexity by eliminating the need for using equalization at the receiver [1]. MIMO (Multiple Input Multiple Output) systems were introduced in $[2,3]$. Under certain conditions [3], the capacity of MIMO systems is shown to increase linearly with $\min \left\{\mathrm{N}_{\mathrm{T}}, \mathrm{N}_{\mathrm{R}}\right\}$ where $\mathrm{N}_{T}$ and $\mathrm{N}_{\mathrm{R}}$ are the number of antennas at the transmitter and the receiver, respectively.

MIMO-OFDM systems provide performance gains because they combine the diversity and multiplexing gains of MIMO with the resilience of OFDM against multi-path fading. In order to achieve these performance improvements, accurate CSI (Channel State Information) is required at the receiver which is obtained via channel estimation. A number of different pilot assisted methods have been proposed in the literature to estimate the channel, such as [4-6].

This paper proposes a pilot-symbol-assisted channel estimation technique for MIMO $2 \times 2$ architecture by assigning on-off pilot symbols between different transmitting antennas. The mixed transmitted signals could be completely separated at the receiver end. Results from simulation shows that the overall system performance is able to be further enhanced.

The paper is organized as follows: firstly, a brief description of the system is provided in Section II. The direct inserted and extracted pilot method is shown in section III. Simulation results are presented in section IV. Finally section $\mathrm{V}$ provides a short discussion and conclusions.

\section{SYSTEM DESCRIPTION}

Fig. 1 shows the model of STBC MIMO-OFDM system. The input of the system is a serial of binary data, mapped onto the M-ary QAM signal 
constellation to give a stream of complex symbols assumed statistically independent. This complex symbol stream came to the STBC encoder to be separated into two independence signals, assume $t_{1}(t)$ and $t_{2}(t)$. Each $t_{i}(t)$ signal is applied to OFDM modulation block. In the OFDM block, the stream is serial-to-parallel converted to produce $a^{n}$ sequence $c_{i, k}$ with $\mathrm{i}=1,2 . \quad c_{i, k}$ is transformed by a inverse fast Fourier transform (IFFT) unit. A guard interval called cyclic prefix (CP) with length $T_{g}$ is added to this signal, yielding a $T$-spaced discrete-time representation of the transmitted signal. The $n^{\text {th }}$ transmitted OFDM block is given by:

$$
s_{i, n}(t)=\frac{1}{\sqrt{N}} \sum_{k=0}^{N-1} c_{i, k} \phi_{n}(t-n T)
$$

Where

$$
\phi_{i, k}(t)= \begin{cases}\exp \left(j \cdot 2 \pi \cdot f_{k} \cdot t\right), & \forall t \in\left[-T_{g}, T\right] \\ 0 & \text { othersiwe }\end{cases}
$$

Where $\mathrm{N}$ is the number of the subcarriers. $f_{k}=f_{0}+\frac{k}{T_{u}}$ and $f_{0}=0, i=1,2$.

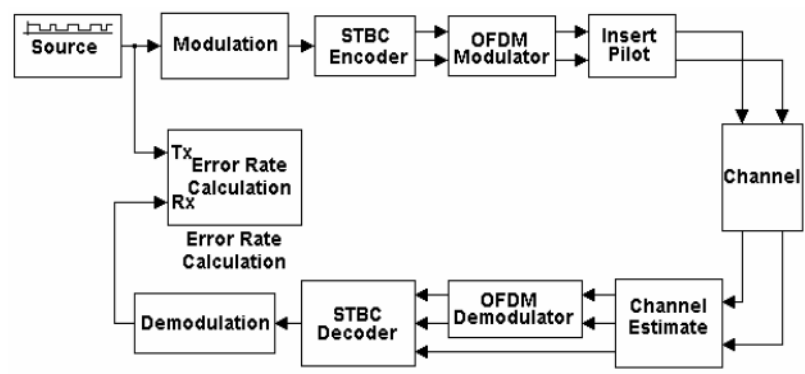

Figure. 1. STBC MIMO-OFDM system with pilot-aided method

If the signal $s(t)=A \cos 2 \pi f_{c} t$ is transmitted over a multipath fading channel, the output is given by:

$$
y(t)=A \sum_{i=1}^{N} a_{i} \cos \left(2 \pi f_{c} t+\theta_{i}\right)+n(t)
$$

Where $a_{i}$ is the attenuation and $\theta_{i}$ is the phase shift of the $i^{\text {th }}$ multipath component. We know that $a_{i}$ and $\theta_{i}$ are random variable. $n(t)$ is complex additive white
Gaussian noise with two-sided spectral density $N_{0} / 2$.

If $N$ is large, the received signal can be rewritten as:

$$
y(t)=A R(t) \cos \left(2 \pi f_{c} t+\theta(t)\right)+n(t)
$$

where $R(t)$ has a Rayleigh distribution.

The amplitude distortion $\mathrm{R}(\mathrm{t})$ can severely degrade performance of wireless systems operating in a fading channel. The relation between the Signal to Noise Ratio (SNR) and Bit Error Rate (BER) in the cases that the channel affected by Additive White Gaussian Noise (AWGN) and fading plus AWGN show clearly how the BER be degraded by fading distortion.

At the receiver, the received signal is passed through a receiver filter and then sampled. The data samples are serial to parallel converted, and applied to the remove guard and FFT processor. The guard interval is removed and only the data in the time interval $[0, T]$ is employed and the output signal is converted back to a serial data sequence and demodulated.

\section{CHANNEL ESTIMATION}

For a $2 \times 2$ STBC system, the channel at time $t$ can be modelled with assumption that fading is constant across two consecutive symbols as:

$$
h_{i}(t)=h_{i}(t+T)=\alpha_{i} e^{j \theta_{i}} \quad i=0,1,2,3
$$

where $T$ is the symbol duration.

STC proposed by Alamouti [7] can be expressed as shown in Table 1:

TABLE 1. Transmitted and received signal at antennas

\begin{tabular}{|l|l|l|l|l|}
\hline & $\begin{array}{l}\text { Antenna } \\
0\end{array}$ & $\begin{array}{l}\text { Antenna } \\
1\end{array}$ & $\begin{array}{l}\text { Antenna } \\
\mathrm{Rx} 0\end{array}$ & $\begin{array}{l}\text { Antenna } \\
\mathrm{Rx} \text { 1 }\end{array}$ \\
\hline Time $t$ & $\mathrm{~s}_{0}$ & $\mathrm{~s}_{1}$ & $\mathrm{r}_{0}$ & $\mathrm{r}_{2}$ \\
\hline Time $t+T$ & $-\mathrm{s}_{1}{ }^{*}$ & $\mathrm{~s}_{0}{ }^{*}$ & $\mathrm{r}_{1}$ & $\mathrm{r}_{3}$ \\
\hline
\end{tabular}

The received signal at the two receives antennas can be expressed as: 


$$
\begin{aligned}
& r_{0}=h_{0} s_{0}+h_{1} s_{1}+n_{0} \\
& r_{1}=-h_{0} s_{1}^{*}+h_{1} s_{0}^{*}+n_{1} \\
& r_{2}=h_{2} s_{0}+h_{3} s_{1}+n_{2} \\
& r_{3}=-h_{2} s_{1}^{*}+h_{3} s_{0}^{*}+n_{3}
\end{aligned}
$$

where $n_{i}(\mathrm{i}=0,1,2,3)$ are AWGN noises.

The principle of our channel estimation technique using on-off pilot is shown in Fig. 2. In the OFDM modulation, at first, a fixed number of pilots are introduced into the data frame. In first branch, two onoff pilot symbols [p 0] were inserted in pilot frame. Similarly, we have the pilot $\left[0-\mathrm{p}^{*}\right]$ in the second branch. The on-off condition shows that there is only one channel parameter can be received at receiver at one time. The ratio between the numbers of pilot and numbers of data symbol depend on the channel conditional. In our work, we used two pilots per eight data symbols in transmitted frame. At the ODFM demodulation block, this noisy pilot bit is spitted and fed to channel estimation block in order to determine the information about the channel characteristics.
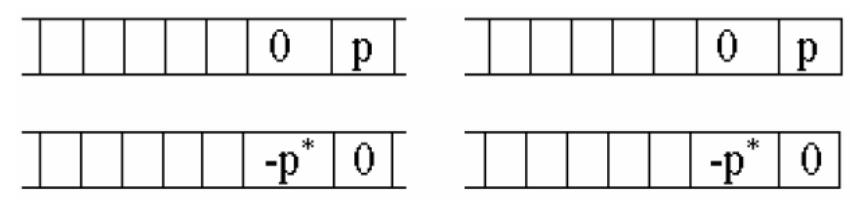

Figure. 2. Channel estimation using on-off pilot

In the receiver, we can obtain the signal at the two antennas as:

$$
\begin{aligned}
& p_{0}=h_{1} p+n_{0} \\
& p_{1}=-h_{0} p^{*}+n_{1} \\
& p_{2}=h_{3} p+n_{2} \\
& p_{3}=-h_{2} p^{*}+n_{3}
\end{aligned}
$$

Thus, we can simply estimate the channel parameters as:

$$
\hat{h}_{0} \approx-\frac{p_{1}}{p^{*}} \hat{h}_{1} \approx \frac{p_{0}}{p} \quad \hat{h}_{2} \approx-\frac{p_{3}}{p^{*}} \hat{h}_{3} \approx \frac{p_{2}}{p}
$$

In the simplest case, we choose $p=1+O i$, we can rewrite the Equ. (8) as:

$$
\widehat{h}_{0}=-p_{1} \quad \widehat{h}_{1}=p_{0} \quad \widehat{h}_{2}=-p_{3} \quad \widehat{h}_{3}=p_{2}
$$

with assumption that noise and pilots are not correlative.

Consequently, we have the channel state information to be used in Space-time block decoder.

\section{SIMULATION RESULTS}

The simulations are carried out for a OFDM system with 144 subscribers and 16-ary QAM constellation on each sub-carrier. The bandwidth of the system is $18 \mathrm{MHz}$ and the FFT-length is 64 . The fading channel is characterized by the maximum Doppler shift of 113

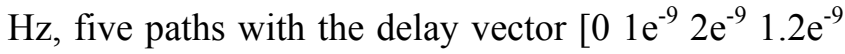
$\left.4 \mathrm{e}^{-9}\right]$ (in seconds) and with the vector gain $[0-5-3-2-$ 3.5] (in $\mathrm{dB}$ ) [8]. Perfect time synchronization is assumed.

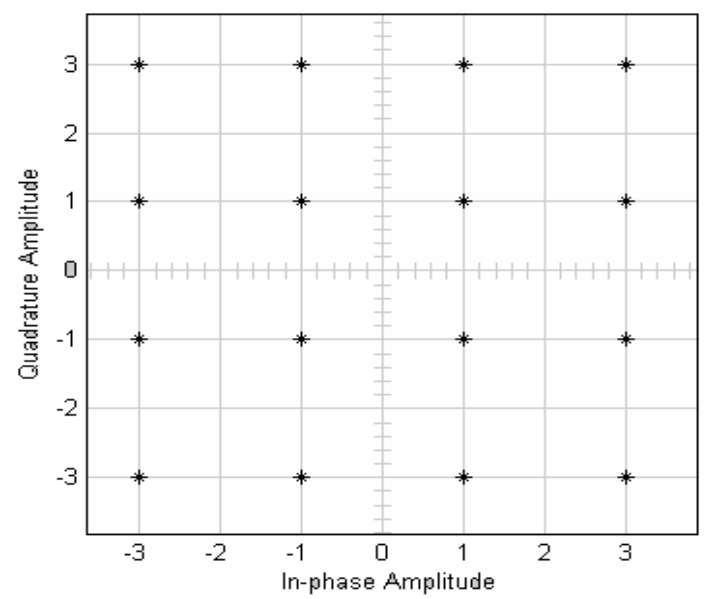

Figure 3. Received 16-ary QAM constellation without any impairment

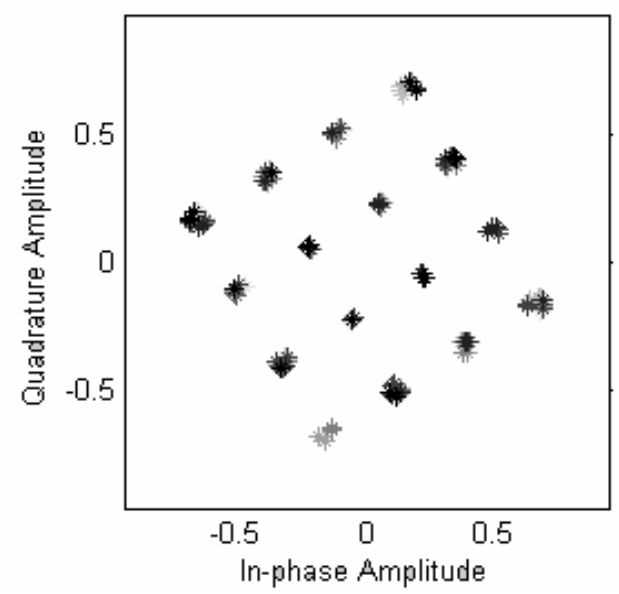

Figure 4. A snap shot of the received 16- QAM constellation with multipath fading 
The effects of impairments on the received 16-ary QAM constellations are illustrated in Figs. 3, 4 and 5, which correspond to the ideal situation (noiseless and without ISI), fading plus AWGN, and fading plus AWGN with pilot assistance. In the ideal case, there are 16 well-defined points. In presence of impairments, the received cloud is due to AWGN and the fading phenomenon.

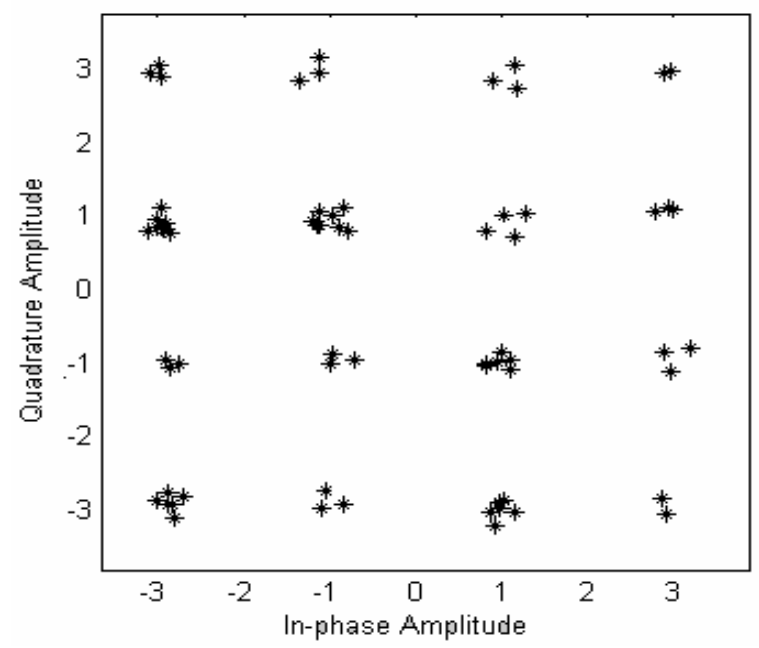

Figure. 5. Received 16-ary QAM constellation with AWGN plus multipath fading after the Equalizer block

The BER in term of SNR, varying between 0 and $10 \mathrm{~dB}$ of this model is shown in Fig. 6 within 4 cases listed in Table 2. The BER performance of the proposed system is evaluated using the Monte-Carlo method.

TABLE 2. Four schemes in the proposed OFDM system

\begin{tabular}{|c|l|l|}
\hline $\begin{array}{c}\text { Scheme } \\
\text { No. }\end{array}$ & \multicolumn{1}{|c|}{ Impairments } & \multicolumn{1}{|c|}{$\begin{array}{c}\text { Compensation } \\
\text { method }\end{array}$} \\
\hline 1 & Rayleigh + AWGN noise & $\begin{array}{l}\text { No- EQ in SISO } \\
\text { system }\end{array}$ \\
\hline 2 & Rayleigh + AWGN noise & EQ in SISO system \\
\hline 3 & Rayleigh + AWGN noise & $\begin{array}{l}\text { No- EQ in MIMO } \\
\text { system }\end{array}$ \\
\hline 4 & Rayleigh + AWGN noise & $\begin{array}{l}\text { EQ in MIMO } \\
\text { system }\end{array}$ \\
\hline
\end{tabular}

The curves show the connection between BER and SNR in four scenarios respectively. Comparing the results of the $1^{\text {st }}$ scenario versus the $2^{\text {nd }}$, the serious influence of the fading is evidenced. By using the pilot-assisted channel estimation, the scheme (2) had over $6 \mathrm{~dB}$ higher SNR gain at the BER of $10^{-2}$ than the existing schemes (4).

Comparing the results between the SISO and MIMO systems, it is evidenced that MIMO-OFDM systems can improve the BER performance in both two cases: with pilot-assistance or without pilotassistance. With the pilot insertion method, MIMO OFDM system had over $3 \mathrm{~dB}$ higher SNR gain at the BER of $10^{-5}$ than the existing SISO system.

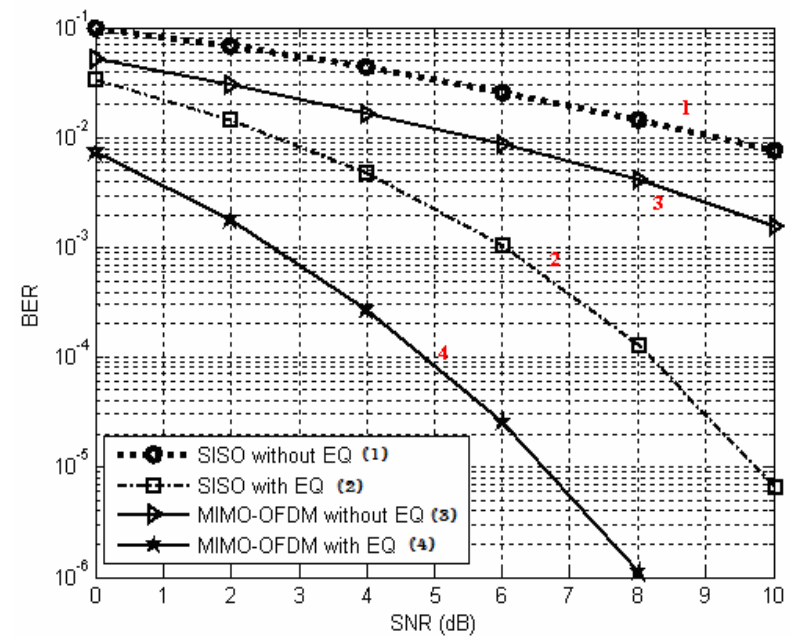

Figure. 6. The plot of BER vs SNR offour scenarios

\section{CONCLUSIONS}

Wireless communication networks often require high quality of sound and the high rate. Moreover, the physical size of mobile devices becomes smaller and their performance needs to be robust in various environments. The inherent problem of fading is always a major impairment of the wireless communication channel. In this paper, the simple and effective method are proposed to estimate CSI over multipath fading channel. Results show that this simple technique tremendously enhances the performance of the compensated system. The scheme proposed in this paper in shown to enjoy the effect.

\section{ACKNOWLEDGMENT}

This work is supported by the QC-10 project of Coltech, VNUH. 


\section{REFERENCES}

[1] R. V. Nee, and R. Prasad, "OFDM for Wireless Multimedia Communications", Artech House Publishers, 1999.

[2] I. E. Telatar, "Capacity of Multi-Antenna Gaussian Channels”, Europ. Trans.Telecomm., vol. 10, pp 585$595,1999$.

[3] G. J. Foschini and M. J. Gans, "On Limits of Wireless Communications in a Fading Environment When Using Multiple Antennas", Wireless Personal Comm., vol. 6, pp 311-335, 1998.

[4] H. Minn and N. Al-Dhahir, "Optimal Training Signals for MIMO OFDM Channel Estimation”, IEEE Trans. Wireless Comm., vol. 5, pp 1158-1168, 2006.

[5] L. Huang, C. K. Ho, J. W. M. Bergmans and F. M. J. Willems, "Pilot-Aided Angle-Domain Channel Estimation Techniques for MIMO-OFDM Systems", IEEE Trans. Vehicular. Tech., vol. 57, no. 2, pp 906920, 2008.

[6] Y. Qiao, S. Yu, P. Su and L. Zhang, "Research on an Iterative Algorithm of LS Channel Estimation in MIMO OFDM Systems", IEEE Trans. Broad., vol. 51, no. 1, pp 149-153, 2005.

[7] SM Alamouti, "A simple transmit diversity technique for wireless communications", IEEE Journal on selected areas in communications, pp. 1451-1458,1998.

[8] T. D. Tan, N. V. Tung, N. N. Thanh, V. V. Hung, H. H. Tue, "Performance of OFDM Using Digital Adaptive Pre-Distorter and Equalizer Over Multipath Fading Channels”, REV-2006, Hanoi, 2006, pp. 76-80, 2006.

\section{AUTHORS' BIOGRAPHIES}

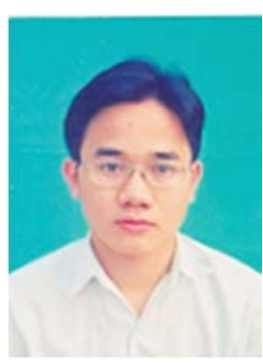

Tran Duc Tan was born in $1980 . \mathrm{He}$ received his B.Sc and M.Sc degrees respectively in 2002 and in 2005, both at the College of Technology (COLTECH), Vietnam National University - Hanoi, Vietnam (VNU), where he has been a lecturer since 2006. $\mathrm{He}$ is currently completing his $\mathrm{PhD}$ thesis at COLTECH, VNUH. He is author and coauthor of several papers on MEMS based sensors and their application. His present research interest is in DSP for communication.

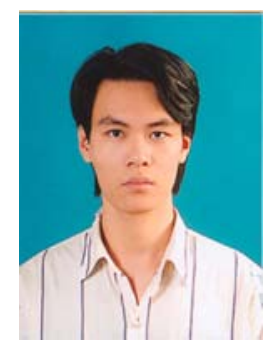

Ta Duc Tuyen was born in $1986 . \mathrm{He}$ received the B.S. degree (Hon.) in electrical engineering from College of Technology (COLTECH), Vietnam National University - Hanoi, Vietnam, where he is currently working toward the M.S. degree. His research interests include digital signal processing techniques for MIMO and MIMO-OFDM system.

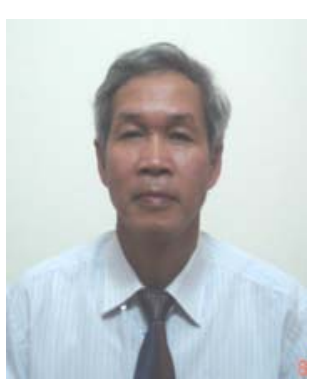

Trinh Anh Vu graduated Bachelor of Radio Physics in 1983 in Hanoi University, Vietnam. He got the $\mathrm{PhD}$ of Radio Physics in 1994 in MG University, Russian Federation. From 1996 to now, he has been working in College of Technology, VNU-Hanoi. From 2007 to 06/2009, Dr. Trinh Anh $\mathrm{Vu}$ is the Dean of Electronics and Telecommunications Faculty, Coltech. His interested research is about wireless communication, MIMO technology and Wimax as well.

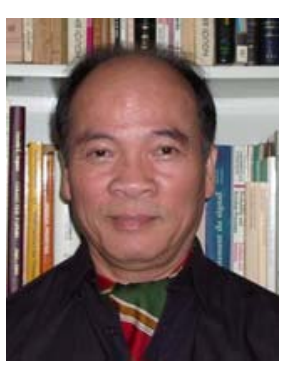

Huu Tue Huynh received his Sc.D. from Laval University in 1972, where he had been a Professor of the Department Electrical and Computer Engineering since 1969. He left Laval in 2004 to become the Chairman of the Department of Information Processing of the College of Technology, Vietnam National University, Hanoi and recently nominated Rector of $\mathrm{Bac} \mathrm{Ha}$ International University. He is author and coauthor of more than one hundred papers published in professional journals and international conferences; he is also coauthor of two books. His research interests cover stochastic simulation techniques, information processing, fast algorithms and architectures with applications to digital communications 